of reported devices are caused by impurities or defects in the systems, rather than by the molecular species under study. Song et al. ${ }^{6}$ have avoided such uncertainties by thoroughly characterizing the charge-transport properties of their devices using a combination of spectroscopy techniques in situ. In this way, they provide unprecedented insight into the underlying physics of charge transport in their molecular transistors.

The authors used inelastic electron tunnelling (IET) spectroscopy to measure the interactions between the tunnelling electrons and the vibrational modes of the molecules in their devices. This technique provides definitive proof that the measured currents actually pass through the molecules in single-molecule transistors, and yields some information about the pathways taken by electrons as they cross the junctions ${ }^{8}$. The authors tested two types of transistor, each with a different molecule in the junction - either an alkane dithiol (which contains two SH groups connected by a saturated hydrocarbon chain) or an aromatic dithiol (which contains two SH groups connected by a benzene ring). Because each dithiol has its own vibrational 'fingerprint', the IET spectra of the devices provide unambiguous evidence of the molecules in the junctions.

The second technique used by Song et al. was transition-voltage spectroscopy. Electrons crossing a molecular junction do so using one of two tunnelling mechanisms that depend on the magnitude of the source-drain voltage; the transition voltage $\left(V_{\text {trans }}\right)$ is the voltage at which tunnelling switches from one mechanism to the other. It has previously been shown ${ }^{9}$ that $V_{\text {trans }}$ is proportional to the difference in energy between the gating orbital of the molecular junction (the orbital that modulates electron tunnelling) and the Fermi levels of the source and drain electrodes, where the Fermi level is the highest possible energy for a conducting electron in an electrode. By measuring $V_{\text {trans }}$ using transitionvoltage spectroscopy at different applied gate voltages, Song et al. demonstrated that a linear relationship exists between gate voltage and molecular-orbital energy in their devices, as expected for single-molecule transistors.

The nature of the molecular orbital that couples to the tunnelling electrons (that is, whether or not the orbital is occupied by electrons from the molecule in the junction) can be determined from the change in conductance of the transistor with respect to the applied gate voltage. Song et al. found that both of their transistor types become more conducting when a negative gate voltage is applied. Because negative gate voltages lower the energy difference between the highest occupied molecular orbital (HOMO) of the molecular junction and the electrode's Fermi level, this indicates that, in their device, tunnelling electrons couple to the HOMOs of the molecules.

The authors found further evidence that the current through their device was gated by the HOMO energy of the molecular junction by a

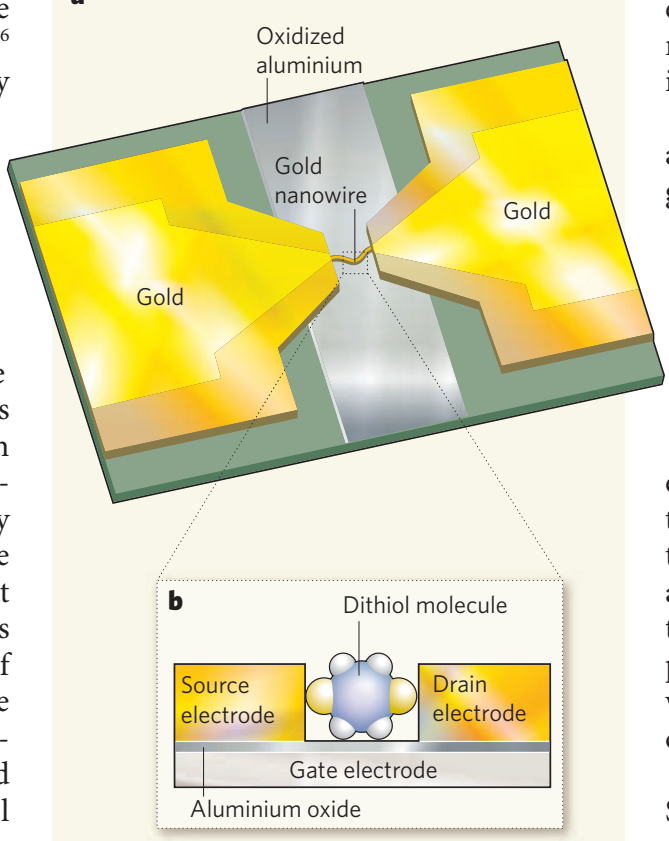

Figure 1 | Single-molecule transistors. Song et $a{ }^{6}{ }^{6}$ have made and characterized singlemolecule transistors in which current flow is controlled by electrostatically modulating the energy of the molecular orbitals of a single molecule. a, Each device consists of a fractured gold nanowire overlaid on a strip of oxidized aluminium. b, Side-on, close-up view of a device. The broken ends of the nanowire form the source and drain electrodes of the transistor, and the oxidized aluminium forms the gate electrode. Aluminium oxide on the surface of the gate electrode provides a necessary layer of insulating material known as the gate dielectric. A single molecule (here, an aromatic dithiol) connects the source and drain electrodes. The electric field created by the gate electrode modulates the energies of molecular orbitals in the dithiol, which in turn control the amount of current that flows through the source and drain electrodes. The components of the device are not drawn to scale.

examining the dependence of the IET spectra on the applied gate voltage. The IET spectra of the transistors that incorporate alkane dithiols were essentially unaffected by the gate voltage. This indicates that electron tunnelling through the device is always 'non-resonant', that is, there is a large energy difference between the dithiol's $\mathrm{HOMO}$ and the electrode's Fermi level.

Conversely, Song et al. observed that the applied gate voltage strongly modulates the IET spectra of transistors that incorporate an aromatic dithiol. Specifically, when a negative gate voltage is applied (which brings the energy of the molecular junction's HOMO closer to that of the electrode's Fermi level), the signal intensities of the spectra increase greatly and the shapes of the vibrational peaks change. The change in peak shape is a clear indication of increased coupling between the tunnelling charge carrier and the molecular vibrations, owing to a resonance between the HOMO and the Fermi level ${ }^{10}$. The authors have thus provided the first experimental demonstration that resonant and non-resonant vibrational coupling can be tuned in single-molecule transistors.

One of the most surprising features of Song and co-workers' study ${ }^{6}$ is the strong effect of the gate voltage on the molecular-orbital energy of their device. For both dithiols studied, the molecular orbitals shifted in energy by 0.25 electronvolts when 1 volt was applied to the gate electrode, a remarkably strong coupling. It is unclear why the gate coupling should be so strong, but the most

likely explanation is that the molecules are extremely close to, or in intimate contact with, the gate dielectric (the oxidized aluminium of the gate electrode). The need for such precise alignment may in part explain why so few of the devices prepared by the authors functioned properly as transistors - only 35 out of 418 were found to have the desired current-voltage characteristics.

Through their multi-spectroscopy approach, Song et. al. ${ }^{6}$ have provided the first conclusive evidence that a solid-state molecular transistor can function through the relative alignment of its orbital energies with the electrode's Fermi level, and that this alignment can be efficiently tuned by the applied gate voltage. Their work sets a benchmark for the validation of future studies of charge transport in molecular systems. But much work remains to be done before molecular electronic devices can effectively compete with their larger silicon-based brethren. For example, a fabrication method that provides high yields of densely packed singlemolecule devices has yet to be developed. In the meantime, Song and colleagues' work provides an excellent foundation for further development of well-characterized molecular devices. James Kushmerick is at the National Institute of Standards and Technology, Gaithersburg, Maryland 20899, USA.

e-mail: james.kushmerick@nist.gov

1. Park, J. et al. Nature 417, 722-725 (2002).

2. Liang, W., Shores, M. P., Bockrath, M., Long, J. R. \& Park, H. Nature 417, 725-729 (2002).

3. Kubatkin, S. et al. Nature 425, 698-701 (2003).

4. Yu, L. H. \& Natelson, D. Nano Lett. 4, 79-83 (2004).

5. Ghosh, A. W., Rakshit, T. \& Datta, S. Nano Lett. 4, 565-568 (2004).

6. Song, H. et al. Nature $462,1039-1043$ (2009).

7. Xu, B. \& Tao, N. J. Science 301, 1221-1223 (2003)

8. Troisi, A. et al. Proc. Natl Acad. Sci. USA 104, 14255-14259 (2007).

9. Beebe, J. M., Kim, B.-S., Gadzuk, J. W., Frisbie, C. D. \& Kushmerick, J. G. Phys. Rev. Lett. 97, 026801 (2006)

10. Galperin, M., Ratner, M. A. \& Nitzan, A. J. Chem. Phys. 121, 11965-11979 (2004).

\section{Word of the Year}

The News \& Views WotY for 2009 is 'critical'. But this is not an accolade. This devilish little word has an honourable place in the scientific lexicon when bound together with such terms as 'mass', 'angle' and 'temperature'. But in an evolutionary battle to convey the sense of 'having a decisive importance', it has all but killed off such alternatives as 'crucial', 'vital', 'essential' and 'necessary'. We can but hope that its spread is halted in 2010. 